\title{
Effective spin-orbit models using correlated first-principles wave functions
}

\author{
Yueqing Chang and Lucas K. Wagner (D) \\ Department of Physics, University of Illinois at Urbana-Champaign, Urbana, Illinois 61801, USA
}

(Received 21 September 2018; revised manuscript received 8 July 2019; accepted 24 December 2019; published 24 February 2020)

\begin{abstract}
Diffusion Monte Carlo using continuous real-space wave functions is one of the most accurate scalable manybody methods for solid-state systems. However, to date, spin-orbit interactions have not been incorporated into large-scale calculations at a first-principles level, only having been applied to small systems. In this technique, we use explicitly correlated first-principles diffusion Monte Carlo calculations to derive an effective spin-orbit model Hamiltonian. The simplified model Hamiltonian is then solved to obtain the energetics of the system. To demonstrate this method, benchmark studies are performed in main-group atoms and monolayer tungsten disulfide, where high accuracy is obtained.
\end{abstract}

DOI: 10.1103/PhysRevResearch.2.013195

\section{INTRODUCTION}

The interplay between the electron correlation and relativistic effects gives rise to a plethora of intriguing quantum phases in condensed-matter systems, including the proposed axion insulating state, topological Mott insulating state, Weyl semimetal, and quantum spin liquid [1,2]. The spin-orbit effect can remove orbital degeneracies and modify the electronic and magnetic structures of materials. In numerical studies, it has been widely investigated in the weakly interacting regime using mean-field theory, which has resulted in the prediction of topological phases [3-5]. In recent years, growing attention has turned to its effect in the strong-to-intermediate correlation regime, where the spin-orbit interactions have been proposed to play important roles in unconventional Mott insulating and topological semimetal states [6,7], quantum spin liquid [8,9], axion insulating state [10], and multipolar orders $[11,12]$. The detailed mechanism and a clear physical picture of how the electron correlation and the spin-orbit interaction cooperate to generate these phenomena remain unresolved in first-principles numerical simulations.

In first-principles calculations, it is a major challenge to simultaneously describe both electron correlation and spin-orbit effects in materials. Many-body wave-function techniques are the most direct way of accessing electron correlations with high accuracy. Previous work has mainly focused on including the spin-orbit interaction in the post-Hartree-Fock methods such as multiconfigurational self-consistent field [13], configuration interaction singles and doubles [14], and coupled cluster [15]. Due to the scaling of these deterministic methods with the system size, calculations can be very expensive when applied to extended systems. The electron correlation

Published by the American Physical Society under the terms of the Creative Commons Attribution 4.0 International license. Further distribution of this work must maintain attribution to the author(s) and the published article's title, journal citation, and DOI. contributions, which are mainly from doubly and higher excitations, are slowly convergent, while the spin-orbit interaction is dominated by the singly excited configurations [16]. Implementation of a Hamiltonian with Rashba-type spin-orbit coupling with auxiliary-field quantum Monte Carlo was also presented and applied to cold atoms [17].

Among the very many first-principles methods, in particular, quantum Monte Carlo techniques using many-body wave functions in the continuum have seen success [18-21] in computing the properties of materials with strong correlation. Here we address first-principles quantum Monte Carlo calculations on bulk materials that also include spin-orbit effects.

Spin-orbit effects combined with electron correlation are commonly considered for small molecular systems. Expansion of the spin-orbit interaction operator in terms of configuration-state functions suffer from a slow convergence of the electron correlation contribution [16]. As for methods based on stochastic sampling procedures, a recent work employed both an auxiliary continuous parameter to represent the electron spin in the wave function and a modified sampling process in the quantum Monte Carlo (QMC) calculation [22-24]. This method is shown to obtain accurate results for atoms and few-atom molecules, two-dimensional homogeneous electron gas, quantum wells, and circular quantum dots with Rashba interaction [25-27]. However, since the spin-orbit energy and the total Coulomb interaction energy are treated on the same footing (a positive feature for some reasons), the computational cost needed to resolve the tiny energy differences among different spin configurations can be very high for solid-state systems.

In this paper, we demonstrate a method for treating spinorbit interactions using quantum Monte Carlo in a scalable and accurate way through rigorous model Hamiltonians. We implement spin-orbit interaction calculations using samples of static-spin many-body wave functions, then downfold this set of first-principles data to a model Hamiltonian. These wave functions are obtained using diffusion Monte Carlo, where the electron-electron interaction is included explicitly as the Coulomb interaction in the many-body Hamiltonian. 
The wave functions are sampled such that they are on a manifold where the only energy difference is contributed by the spin-orbit interaction. Within this context, the computational cost needed to resolve different spin configurations is greatly reduced compared to dynamical spins in the calculation, very similar to that needed in a standard fixednode diffusion Monte Carlo (FN-DMC) calculation (the fixedphase approximation is used for complex wave functions). To demonstrate our method, we perform benchmark studies in small atomic systems and a periodic solid-state system. Using this technique, we calculate the ground-state fine-structure splittings induced by spin-orbit interaction for main-group atoms. Our calculations generate values that agree with the experiments for most atoms, with errors attributed mainly to orbital relaxations in the mean-field calculations. To show that this method can be easily generalized to realistic materials, we compute the band splitting at the $K$ point for monolayer tungsten disulfide $\left(\mathrm{WS}_{2}\right)$, yielding a value of $0.39(2) \mathrm{eV}$, as compared to the photoluminescence measurement of $0.4 \mathrm{eV}$ [28].

\section{EFFECTIVE SPIN-ORBIT HAMILTONIAN}

Our method is quite general and can treat effective interactions as well as multiple bands $[29,30]$. However, for this paper, we will focus on the case of electronic states that are degenerate in the absence of spin-orbit interaction; for example, the six occupations of a single electron on a $p$ manifold. Consider many-body wave function $\left|\Psi_{N}\right\rangle$ within that degenerate subspace. Then, the expectation value of the energy is given by

$$
\left\langle\Psi_{n}\left|\hat{H}_{\text {rel }}\right| \Psi_{n}\right\rangle=E_{0}+E_{\text {SOI }}\left[\Psi_{n}\right],
$$

where we evaluate $E_{\text {SOI }}$ by subtracting an averaged-relativistic effective core potential (ARECP) from the relativistic effective core potential (RECP) [24]:

$$
\begin{aligned}
\widehat{W}_{\text {SOI }}= & \sum_{i=1}^{N_{\text {clec }}} \sum_{I=1}^{N_{\text {ions }}} \sum_{l=0}^{L-1} \frac{2}{2 l+1}\left(\hat{v}_{l, j=l+\frac{1}{2}}^{i I}-\hat{v}_{l, j=l-\frac{1}{2}}^{i I}\right) \\
& \times \sum_{m_{l}=-l}^{l} \sum_{m_{l}^{\prime}=-l}^{l}\left|l m_{l}\right\rangle\left\langle l m_{l} \hat{\mathbf{I}}^{i} \cdot \hat{\mathbf{s}}^{i} \mid l m_{l}^{\prime}\right\rangle\left\langle l m_{l}^{\prime}\right|,
\end{aligned}
$$

where operators $\hat{v}_{l, j=l \pm \frac{1}{2}}^{i I}$ are the spin-up/-down radial parts of the two-component RECP between the $i$ th electron and the $I$ th ion, respectively. The spin-orbit energy in Eq. (1) is given by $E_{\mathrm{SOI}}\left[\Psi_{n}\right]=\left\langle\Psi_{n}\left|\widehat{W}_{\mathrm{SOI}}\right| \Psi_{n}\right\rangle$.

We take the effective Hamiltonian of the degenerate manifold to be

$$
\widehat{H}_{\mathrm{rel}}^{\mathrm{eff}}=E_{0}+\lambda \sum_{i=1}^{N_{\text {elec }}} \sum_{I=1}^{N_{\text {ins }}} \widehat{\mathbf{L}}_{i I} \cdot \widehat{\mathbf{S}}_{i}
$$

where the operator $\widehat{\mathbf{L}}_{i I}=\left(\mathbf{r}_{i}-\mathbf{r}_{I}\right) \times \frac{1}{\mathrm{i}} \nabla_{i}$ is the orbital angular momentum of the $i$ th electron relative to $I$ th ion, and $\mathbf{S}_{i}$ is the spin angular momentum operator acting on the $i$ th electron.

Using the results proven in $[29,30]$, the effective $\lambda$ can be estimated using the first-principles wave functions $\left|\Psi_{n}\right\rangle$ by taking the expectation values on both sides of Eq. (3) with respect to some many-body wave function $\left|\Psi_{n}\right\rangle$,

$$
\left\langle\Psi_{n}\left|\hat{H}_{\mathrm{rel}}\right| \Psi_{n}\right\rangle=E_{0}+\lambda\left\langle\Psi_{n}\left|\sum_{i=1}^{N_{\text {elec }}} \sum_{I=1}^{N_{\text {ions }}} \widehat{\mathbf{L}}_{i I} \cdot \widehat{\mathbf{S}}_{i}\right| \Psi_{n}\right\rangle .
$$

This relationship is true for any wave function $\left|\Psi_{n}\right\rangle$ sampled from the degenerate manifold if the spin-orbit model is valid. Since both $E_{\mathrm{SOI}}\left[\Psi_{n}\right]$ and $\left\langle\Psi_{n}\left|\sum_{i I} \widehat{\mathbf{L}}_{i I} \cdot \widehat{\mathbf{S}}_{i}\right| \Psi_{n}\right\rangle$ can be computed using first-principles wave functions, the coefficient $\lambda$ can be obtained by fitting to the linear relationship between these two values. Using Eq. (1), we thus have

$$
E_{\mathrm{SOI}}\left[\Psi_{n}\right]=\lambda\left\langle\Psi_{n}\left|\sum_{i=1}^{N_{\text {elec }}} \sum_{I=1}^{N_{\text {ions }}} \widehat{\mathbf{L}}_{i I} \cdot \widehat{\mathbf{S}}_{i}\right| \Psi_{n}\right\rangle .
$$

For periodic systems, the effective Hamiltonian at momentum $\mathbf{k}$ is given by $\widehat{H}_{\mathbf{k}, \text { SOI }}^{\text {eff }}=\sum_{i I} \lambda_{I}(\mathbf{k}) \widehat{\mathbf{L}}_{i I} \cdot \widehat{\mathbf{S}}_{i}$ (for the monolayer $\mathrm{WS}_{2}$, the spin-orbit interaction is mostly contributed by $\mathrm{W}$ atoms). This method can be easily extended to the case where the wave functions do not belong to a degenerate manifold by including parameters that represent orbital energies, interaction terms, and so on. This has been performed for non-spin-orbit-coupled systems [30].

\section{DETAILS OF THE CALCULATIONS}

We first construct single Slater determinants from the single-particle orbitals computed using Hartree-Fock or density functional theory (DFT). These Slater determinants are sampled such that they differ only by single-particle orbitals that have the same scalar-relativistic energy. We construct the following Slater-Jastrow trial wave functions,

$$
\Psi_{\mathrm{T}}=e^{U} D^{\uparrow} D^{\downarrow},
$$

where the spins are collinear, chosen to be along the $z$ axis. Determinants $D^{\uparrow}$ and $D^{\downarrow}$ are constructed from occupying the lowest-energy spin-up and spin-down single-particle orbitals, respectively. To explicitly incorporate the electron correlation, the trial wave functions are constructed by multiplying these Slater determinants by Jastrow factors $U$ s, which include up to three-body interactions (see [31]). The Jastrow factors are then optimized by minimizing the variance of the trial scalarrelativistic energy. Finally, we apply fixed-phase DMC to project out the lowest-energy wave function that has the same nodal structure as the corresponding trial wave functions. The scalar-relativistic Hamiltonian we use to evolve the trial wave function in fixed-phase DMC is given by expressing the Coulomb potential using the sum of electron-electron interaction among the valence electrons and the averagedrelativistic effective core potential (ARECP). See Appendix A for the detailed expressions of the ARECP and the total scalarrelativistic Hamiltonian. $T$ moves are used to evaluate the nonlocal effective core potentials during the imaginary-time evolution of the trial wave functions [32]. In order to correct the time-step error introduced by the Trotter product, we extrapolate the result to a zero-time-step limit using a method based on the Bayesian probability distribution described in [33]. All variational Monte Carlo (VMC) and fixed-phase DMC calculations are performed using the open-source quantum Monte Carlo code QWALK [31]. 
Because the $a b$ initio spin-orbit interaction Hamiltonian [Eq. (3)] does not commute with the scalar-relativistic Hamiltonian, we correct the mixed estimator error up to first order by computing the extrapolated estimators given in [34]. We compute the extrapolated estimator values of the spinorbit interaction energies and the corresponding descriptor $\left\langle\sum_{i I} \widehat{\mathbf{L}}_{i I} \cdot \widehat{\mathbf{S}}_{i}\right\rangle$ for several fixed-phase wave functions, then find the coefficient $\lambda$ using linear regression. This procedure maps a set of values calculated from first-principles wave functions to a model Hamiltonian given by Eq. (3). Such procedures can be generalized to expectation values calculated using dynamic-spin wave functions, but for the benchmarking systems considered here, static-spin wave functions are sufficient.

\section{THE STARTING POINTS OF BENCHMARK STUDIES}

For the atomic systems, we use GAMESS $[35,36]$ to generate the single-particle orbitals for the cations and anions $\mathrm{Ca}^{+}$, $\mathrm{In}^{+}, \mathrm{Ge}^{2+}, \mathrm{Sn}^{2+}, \mathrm{Br}^{-}$, and $\left.\mathrm{I}^{-}\right)$, such that the $n p_{x}, n p_{y}$, and $n p_{z}$ orbitals have exactly the same energies for each ion. Thus, we can reduce the orbital relaxation effect introduced by performing restricted open-shell Hartree-Fock calculations directly on atomic systems, Then, we use these ionic orbitals to construct the Slater determinants for the corresponding atoms. We perform the calculations using the Dolg-Stoll and the Trail-Needs ECPs and the available basis sets provided in [37-42]. The Dolg-Stoll ECPs include relativistic effects implicitly by adjusting the orbitals at the multiconfiguration Dirac-Hartree-Fock level and including the Breit interaction [37-40]. We also compare the large-core and small-core Dolg-Stoll ECPs, which exclude or include, respectively, the semicore $(n-1)$ spd shells in the valence shell. The Trail-Needs ECPs are large core only for the elements considered here and are finite at the origin, and thus are particularly suitable for QMC calculations [41,42].

As an input for the QMC calculations for the $\mathrm{WS}_{2}$ benchmark study, we use CRYSTAL17 $[43,44]$ to perform mean-field calculations for monolayer $\mathrm{WS}_{2}$, with the lattice constant $a=3.23 \AA$, and the spacing between two $\mathrm{S}$ atoms in the same unit cell, $d=3.19 \AA$. The molecular orbitals are calculated for the primitive cell by solving the Kohn-Sham equation, where the exchange-correlation interactions are included within the generalized gradient approximation in the Perdew-Burker-Ernzerhof (PBE) form [45]. We use a converged $12 \times 12 k$ mesh for reciprocal lattice vectors. The Dolg-Stoll averaged-relativistic ECP (60 core electrons for $\mathrm{W}$ and 10 core electrons for $\mathrm{S}$ ) is used [46,47]. A truncated Gaussian basis set including up to triple- $\zeta$ functions is used to expand the molecular orbitals for W [46]. For S, we use the starting basis set provided in Ref. [47]. Then we use the calculated molecular orbitals to construct Slater determinants. More details about the many-body wave-function sampling are included in Appendix B.

\section{BENCHMARK STUDIES}

Taking the $\mathrm{Ga}$ atom as an example, the ground-state electron configuration without spin-orbit interaction is [Ar] $3 d^{10} 4 s^{2} 4 p^{1}$ with $L=1, S=\frac{1}{2}\left({ }^{2} P\right.$, sixfold degenerate).

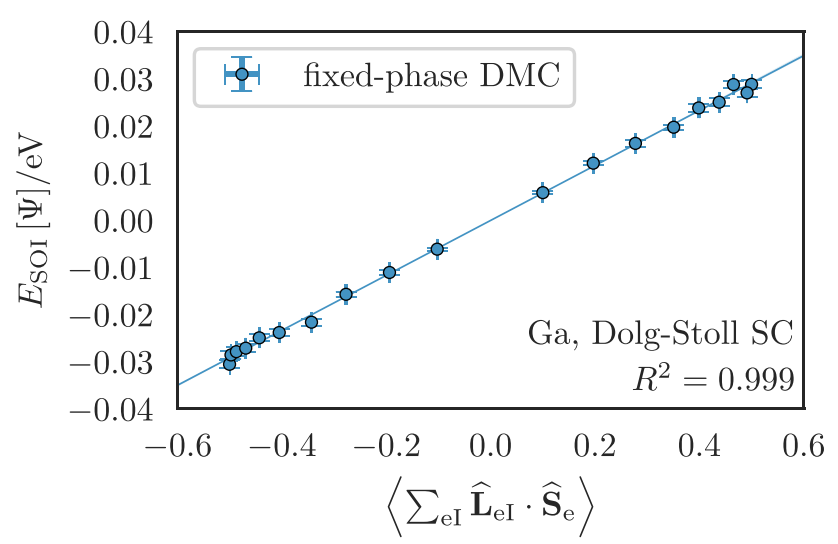

FIG. 1. Calculated spin-orbit interaction energies vs SOI descriptors $\left\langle\sum_{i I} \widehat{\mathbf{L}}_{i I} \cdot \widehat{\mathbf{S}}_{i}\right\rangle$ for 20 wave-function samples for the Ga atom. The Dolg-Stoll small-core ECP is used. The blue circles (fixed-phase DMC) are the fixed-phase DMC extrapolated estimators. The fitted $\lambda_{\text {SOI(DMC) }}$ is $0.058(1) \mathrm{eV}$.

The spin-orbit interaction splits the sixfold degenerate state into two manifolds, the $J=\frac{3}{2}$ quartet and the $J=\frac{1}{2}$ doublet [see Fig. 3(a)]. In the Ga atom, the sampling of wave functions on a sub-Hilbert space with the lowest scalar-relativistic energy is performed by projecting out the fixed-phase wave functions from the trial Slater-Jastrow wave functions, whose Slater parts are linear superpositions of the Slater determinants including $4 p_{x}, 4 p_{y}$, and $4 p_{z}$ orbitals. In our calculations, we generate 20 samples of such trial wave functions with different spin-orbit interaction (SOI) descriptor values $\left\langle\sum_{i I} \widehat{\mathbf{L}}_{i I} \cdot \widehat{\mathbf{S}}_{i}\right\rangle$ ranging from -0.5 to 0.5 in atomic units, when taking the dimensionless $S_{z}$ for spin-up/-down electron to be $\pm 1 / 2$. Within this context, the spin-orbit splitting $\Delta$ of the Ga atom ground-state configuration is given by $\Delta=3 / 2 \lambda$.

In Fig. 1 , we plot the SOI energies $E_{\text {SOI }}$ versus the descriptors $\left\langle\sum_{i I} \widehat{\mathbf{L}}_{i I} \cdot \widehat{\mathbf{S}}_{i}\right\rangle$ calculated using fixed-phase wave functions for $\mathrm{Ga}$ atom. There is a linear relationship between these two quantities, as required by Eq. (4), with the slope identified as the $\lambda$. In Fig. 2, the fitted $\lambda$ using values calculated from fixed-phase DMC, VMC with Slater determinants [VMC(HF)], and VMC with Slater-Jastrow [VMC(SJ)] wave functions are reported for the atomic systems. The fitted $\lambda \mathrm{s}$ obtained using fixed-phase DMC are the closest to the experimental estimates. Note that $\mathrm{VMC}(\mathrm{SJ})$ does not necessarily perform better than VMC(HF) when computing the SOI energies. This behavior is also reported when other nonenergy properties (e.g., the dipole moments [48]) are computed for an optimized wave function.

To demonstrate the accuracy of our technique, we compute the spin-orbit splittings of the ground-state configurations of the following main-group atoms: $\mathrm{Ga}, \mathrm{In}, \mathrm{Ge}, \mathrm{Sn}, \mathrm{Br}$, and I. The $\mathrm{In}, \mathrm{Br}$, and I atom have a similar spin-orbit splitting pattern as $\mathrm{Ga}$, while the ground-state splittings of $\mathrm{Ge}$ and $\mathrm{Sn}$ atoms $\Delta_{J=1}$ and $\Delta_{J=2}$ satisfy $\Delta_{J=1}=1 / 2|\lambda|$ and $\Delta_{J=2}=3 / 2|\lambda|$ [see Fig. 3(a)].

To check if there is dependence on the ECP, we perform the same calculations using the Dolg-Stoll and the TrailNeeds ECPs [37-42]. Figure 3(b) shows the fixed-phase DMC extrapolated estimator values of the SOI splittings 


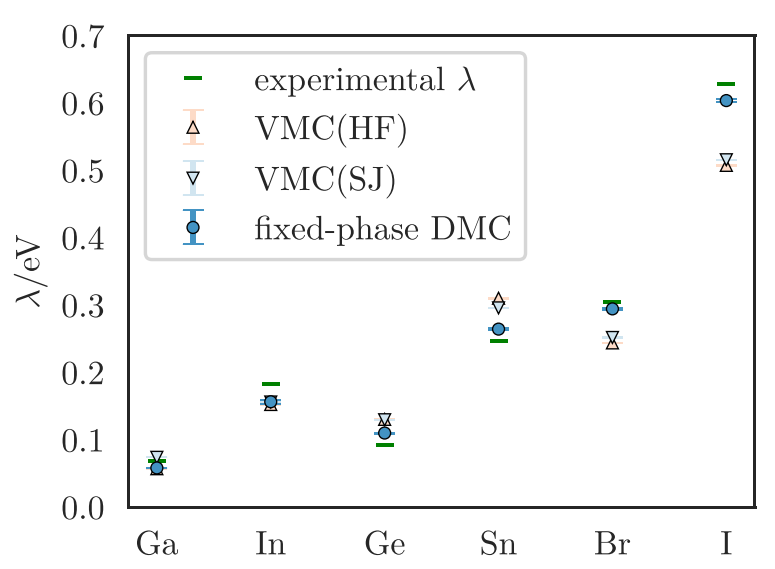

FIG. 2. Fitted $\lambda$ using the results obtained from fixed-phase DMC (circles), VMC with Slater determinants (upper triangles), and Slater-Jastrow wave functions (lower triangles). The experimental estimates are labeled with short horizontal lines. For $\mathrm{Sn}$ and $\mathrm{Ge}$, experimental $\lambda s$ are evaluated as the average of the values obtained from $J=1$ and $J=2$ SOI splittings.

of the ground-state configurations versus the experimentally determined fine-structure splittings for the chosen main-group atoms extracted from the NIST atomic spectra database [49]. The light-gray line indicates an equality between the calculated values and the experimentally determined values. From the figure, we can see that our method yields better agreement with the experiments when using the Dolg-Stoll small-core ECP and the Trail-Needs ECP compared with that when using the Dolg-Stoll large-core ECP.

The discrepancy between the calculated SOI splitting $[0.148(1) \mathrm{eV}]$ and the experimentally determined finestructure splitting $(0.210 \mathrm{eV})$ of the $\mathrm{Sn}$ atom $J=1$ state stems from the fact that the wave functions we sampled are not on the exact lowest-energy subspace of the Hilbert space. This error is first introduced by the orbital relaxation in the mean-field calculations, where the spin-orbit interaction is not included. Generally, the orbital relaxation effect becomes more pronounced when the energy spacing between the $(n+1) p$ and $n p$ manifolds decreases. Moreover, the optimization of the spatial part of the wave functions is performed using only the scalar-relativistic Hamiltonian without the spin-orbit interaction. Therefore, constructed using these orbitals and optimized using the scalar-relativistic Hamiltonian, the sampled many-body wave function is not an exact eigenstate of the fully relativistic Hamiltonian. In order to show this, we perform the Dirac-Hartree-Fock calculation in the DIRAC package using the fully relativistic Dolg-Stoll small-core ECP and basis set. The computed splitting for the Sn atom using Dirac-Hartree-Fock is $\Delta_{J=1}=0.174 \mathrm{eV}$, which is closer to the experimental value.

The effect of the electron correlation on the spin-orbit interaction energy is investigated by performing the same calculation using only the single Slater determinants and the Slater-Jastrow wave functions. For the $\mathrm{Sn}$ atom, where there are two electrons in the $p$ manifold, adding in the Jastrow factor could change the spin-orbit interaction energy for the sampled wave function up to $0.021(2) \mathrm{eV}$ (see Appendix C for more details).

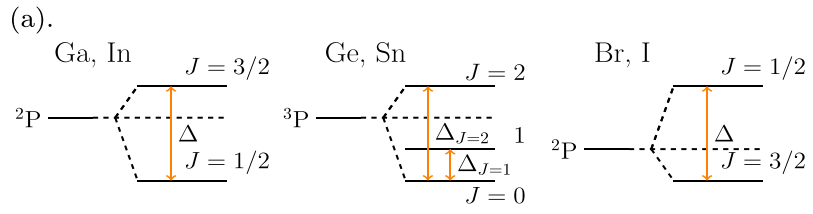

(b).

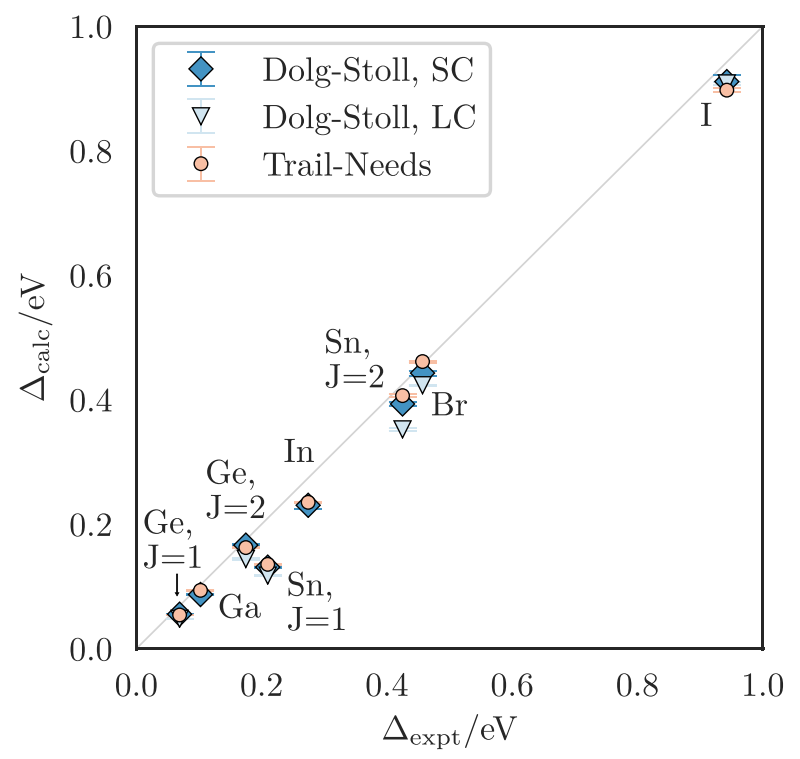

FIG. 3. (a). Spin-orbit splittings of the ground-state electron configurations of $\mathrm{Ga}$, In, Ge, Sn, Br, and I atoms. (b) Fixed-phase DMC extrapolated estimators (the mixed estimators are extrapolated to the zero-time-step limit) vs the experimental values of the fine-structure splittings for the chosen main-group atoms. Different colors and shapes of markers denote calculated values using different ECPs.

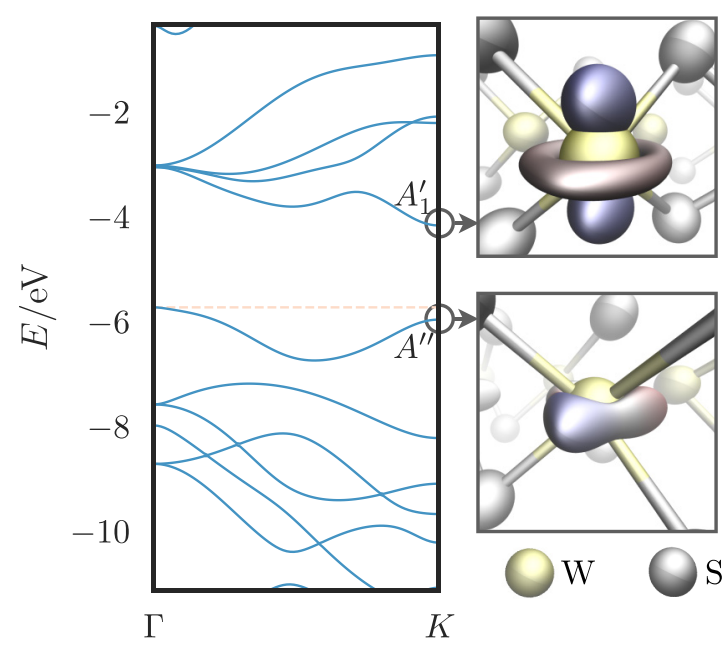

FIG. 4. Band structure of monolayer $\mathrm{WS}_{2}$ calculated on the unit cell, using the Perdew-Burke-Ernzerhof exchange-correlation functional [45] without spin-orbit interaction. The red dashed line represents the Fermi level. The conduction-band minimum and valenceband maximum at $K$ are labeled $A_{1}^{\prime}$ and $A^{\prime \prime}$. The corresponding single-particle orbitals are plotted on the right using the VISUAL MOLECULAR DYNAMICS software [50], with orbital shape derived from the isosurface of the magnitude, colored by the phase. 


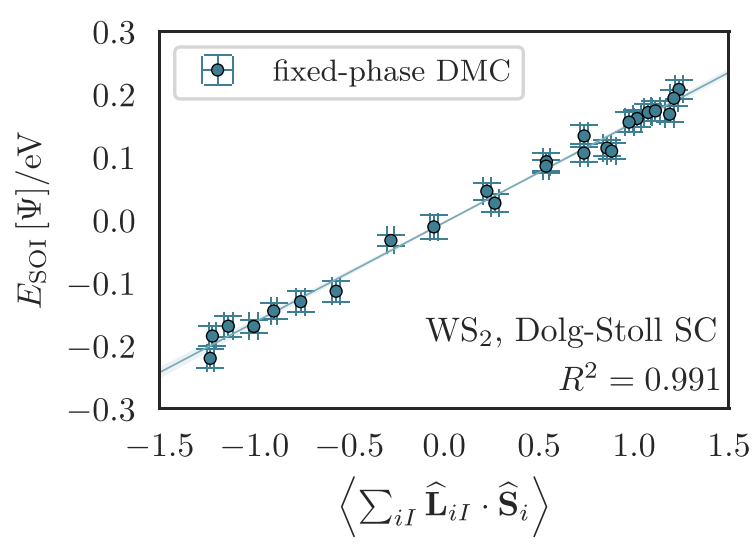

FIG. 5. Calculated spin-orbit interaction energies vs SOI descriptors $\left\langle\sum_{i I} \widehat{\mathbf{L}}_{i I} \cdot \widehat{\mathbf{S}}_{i}\right\rangle$ for 24 fixed-phase wave functions constructed on a manifold that has degenerate scalar-relativistic energy at $K$ for monolayer $\mathrm{WS}_{2}$. The circles are the fixed-phase DMC extrapolated estimators, which are fitted to the solid line. The fitted slope $\lambda_{\text {SOI(DMC) }}$ is $0.159(3) \mathrm{eV}$.

In order to demonstrate that our model can be easily generalized to larger-scale systems, we perform a benchmark study in monolayer $\mathrm{WS}_{2}$. Due to the presence of tungsten atoms, the strong spin-orbit interaction splits the valence-band maximum at $K$. We apply our method to compute this band splitting. We construct trial wave functions from superpositions of four Slater determinants multiplied by three-body Jastrow factors (see Appendix B for more details). These Slater determinants are constructed by promoting one spin-up/-down electron from the twofold degenerate valence-band maximum $\left(A^{\prime \prime}\right)$ to the conduction-band minimum $\left(A_{1}^{\prime}\right)$ at $K$, such that they have degenerate scalar-relativistic energy (see Fig. 4).

Figure 5 shows the fixed-phase DMC extrapolated SOI energies versus the SOI descriptors calculated using samples of many-body wave functions of monolayer $\mathrm{WS}_{2}$ at the $K$ point. The slope of the fitted line is the spin-orbit interaction strength $\lambda$ in our linear model. The calculated spin-orbit splitting $\Delta$ for the valence-band maximum at $K$ is $0.39(2) \mathrm{eV}$. It agrees with the reported band splitting of $0.4 \mathrm{eV}$, determined by measuring the differential reflectance spectra of exfoliated single-layer $2 \mathrm{H}-\mathrm{WS}_{2}$ [28], and the band splitting $0.43 \mathrm{eV}$ previously reported using the $\mathrm{PBE}$ exchange-correlation functional [51]. This demonstrates that our method can be easily generalized to perform spin-orbit interaction calculations in solid-state systems.

\section{CONCLUSION}

We have demonstrated a scalable first-principles quantum Monte Carlo technique for extended systems, using explicitly correlated continuous many-body wave functions; this will allow future computational studies to blend treatment of electron correlation and spin-orbit interactions using QMC calculations. The method is based on deriving an effective Hamiltonian for the spin-orbit interaction from the $a b$ initio energies of sampled many-body wave functions. In this method, the major computational cost is contributed by the evaluation of spin-orbit interaction energy on the fixedphase DMC wave functions, about twice that of a standard fixed-phase DMC calculation (see Appendix E for more details). One can also include electron-electron interactions in the effective Hamiltonian.

We demonstrated this technique in atomic systems and monolayer $\mathrm{WS}_{2}$. For the main-group atoms, we compute the spin-orbit splittings of the ground-state configurations and obtain results in agreement with the experimentally determined fine-structure splittings. Using an optimized Slater-Jastrow wave function changes the spin-orbit interaction energy up to $0.021(2) \mathrm{eV}$ compared with that computed using a single Slater determinant in the $\mathrm{Sn}$ atom.

For the monolayer $\mathrm{WS}_{2}$, we compute the band splitting induced by spin-orbit interaction at $K$. Our first-principles result agrees with previous calculations and experiments, thus demonstrating the ability of this method to be generalized to larger-scale systems. The main error of our calculations stems in that the many-body wave functions are constructed using the orbitals generated from mean-field calculations using a scalar-relativistic Hamiltonian. This will introduce error into the spatial part of the full many-body wave-function samples. Thus, the sampled wave functions might not be on the sub-Hilbert space described by the model Hamiltonian of interest.

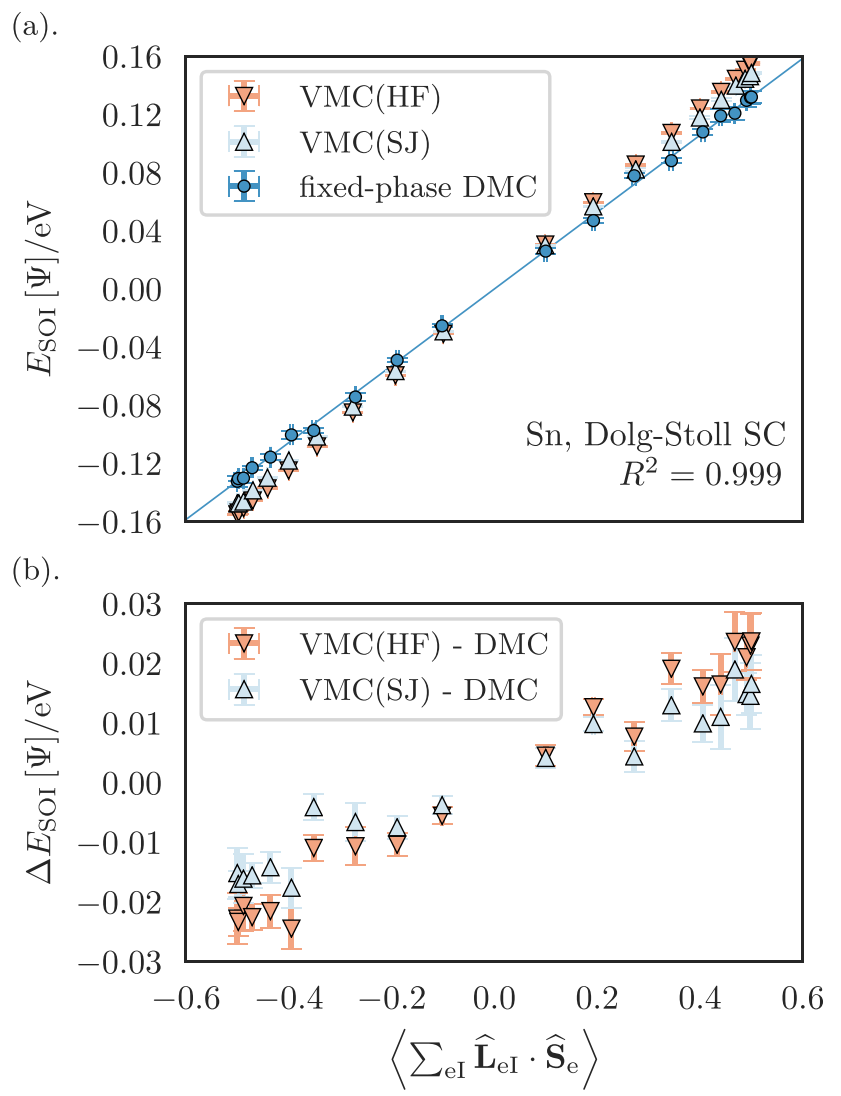

FIG. 6. (a) Calculated spin-orbit interaction energies vs descriptors for the $\mathrm{Sn}$ atom (Stuttgart-Stoll small-core ECP) using VMC, with single Slater determinants (HF) and Slater determinants multiplied by the same three-body Jastrow factor (VMC-SJ), and fixedphase DMC. (b) The differences between the energies calculated using fixed-phase DMC and $\mathrm{VMC}(\mathrm{HF})$ and $\mathrm{VMC}(\mathrm{SJ})$. 


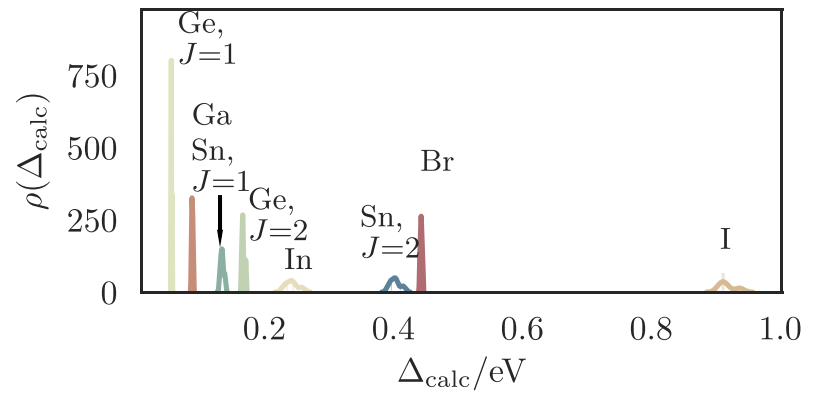

FIG. 7. Normalized distribution of the fixed-phase DMC extrapolated estimators (the DMC mixed estimators are extrapolated to the zero-time-step limit) calculated with available basis sets using the Dolg-Stoll small-core ECPs.

A major promise of this technique is that one can treat electron-electron interactions, spin-orbit effects, and onebody terms in effective Hamiltonians all on the same footing for bulk systems. As mentioned before, the cost is similar to a standard fixed-phase DMC calculation, which will allow it to be applied to realistic models of materials. We envision this opening a new frontier for modeling spin-orbit effects in correlated materials.

\section{ACKNOWLEDGMENTS}

This work was supported by a grant from the Simons Foundation as part of the Simons Collaboration on the Many Electron Problem. The authors would like to thank Cody Melton, Lance Cooper, W. M. C. Foulkes, and Richard M. Martin for helpful discussions.

\section{APPENDIX A: THE AB INITIO SCALAR-RELATIVISTIC HAMILTONIAN}

In order to obtain the correlated first-principles wave function, we evolve the trial wave function in quantum Monte Carlo (QMC) using an ab initio scalar-relativistic Hamiltonian, where the electron-ion Coulomb interaction is written in terms of the averaged-relativistic effective core potential (ARECP) [52], and the electron-electron interaction is explicitly included as the Coulomb interaction,

$$
\begin{aligned}
\widehat{H}_{\text {sca-rel }}= & \sum_{i=1}^{N_{\text {elec }}}-\frac{1}{2 m_{e}} \nabla_{i}^{2}+\sum_{i, j=1, i<j}^{N_{\text {elec }}} \frac{1}{\left|\mathbf{r}_{i}-\mathbf{r}_{j}\right|} \\
& +\sum_{i=1}^{N_{\text {elec }}} \sum_{I=1}^{N_{\text {ion }}} \widehat{W}_{\text {ARECP }}^{i I},
\end{aligned}
$$

where $\widehat{W}_{\text {ARECP }}^{i}=\sum_{l=0}^{L-1} \hat{v}_{l}^{i I} \sum_{m_{l}=-l}^{l}\left|l m_{l}\right\rangle\left\langle l m_{l}\right|+\hat{v}_{L}^{i I}$, and the operators $\hat{v}_{l}^{i I}$ are obtained by averaging over the components with different $j$ s in relativistic effective core potential (RECP): $v_{j}^{i I}(r)=\frac{l+1}{2 l+1} v_{l, j=l+\frac{1}{2}}^{i I}+\frac{l}{2 l+1} v_{l, j=l-\frac{1}{2}}^{i I}(r)$.

\section{APPENDIX B: WAVE-FUNCTION SAMPLING FOR MONOLAYER WS 2 BENCHMARK STUDY}

We sample several many-body trial wave functions with the following configurations:

$$
\Psi(\mathbf{R})=D_{1}+i c D_{2},
$$

\begin{tabular}{|c|c|c|c|}
\hline Benchmark studies & & $\mathrm{ECP}^{\mathrm{a}}$ & Basis set \\
\hline \multirow{7}{*}{ Atoms } & \multirow{2}{*}{$\mathrm{Ga}$} & DS-SC: ECP10MDF & ECP10MDF, VDZ, VTZ, VQZ, V5Z \\
\hline & & TN: Dirac-Fock & $4 s^{2} 4 p^{1}[2 P]$ \\
\hline & In & $\begin{array}{l}\text { DS- SC: ECP28MDF } \\
\text { TN: Dirac-Fock }\end{array}$ & $\begin{array}{c}\text { ECP28MDF, VDZ, VTZ, VQZ, V5Z } \\
5 s^{2} 5 p^{1}[2 P]\end{array}$ \\
\hline & $\mathrm{Ge}$ & $\begin{array}{l}\text { DS-SC: ECP10MDF } \\
\text { DS-LC: ECP28MDF } \\
\text { TN: Dirac-Fock }\end{array}$ & $\begin{array}{c}\text { ECP10MDF, VDZ, VTZ, VQZ, V5Z } \\
\text { ECP28MDF } \\
4 s^{2} 4 p^{2}[3 P]\end{array}$ \\
\hline & $\mathrm{Sn}$ & $\begin{array}{l}\text { DS-SC: ECP28MDF } \\
\text { DS-LC: ECP46MDF } \\
\text { TN: Dirac-Fock }\end{array}$ & $\begin{array}{c}\text { ECP28MDF, VDZ, VTZ, VQZ, V5Z } \\
\text { ECP46MDF } \\
5 s^{2} 5 p^{2}[3 P]\end{array}$ \\
\hline & $\mathrm{Br}$ & $\begin{array}{l}\text { DS-SC: ECP10MDF } \\
\text { DS-LC: ECP28MDF } \\
\text { TN: Dirac-Fock }\end{array}$ & $\begin{array}{l}\text { VDZ, VTZ, VQZ, V5Z } \\
\text { ECP28MDF } \\
4 s^{2} 4 p^{5}[2 P]\end{array}$ \\
\hline & I & $\begin{array}{l}\text { DS-SC: ECP28MDF } \\
\text { DS-SC: ECP46MDF } \\
\text { TN: Dirac-Fock }\end{array}$ & $\begin{array}{l}\text { VDZ, VTZ, VQZ, V5Z } \\
\text { ECP46MDF } \\
5 s^{2} 5 p^{5}[2 P]\end{array}$ \\
\hline Monolayer $\mathrm{WS}_{2}$ & $\begin{array}{l}\mathrm{W} \\
\mathrm{S}\end{array}$ & $\begin{array}{l}\text { DS-SC: ECP60MDF } \\
\text { DS-SC: ECP10SDF }\end{array}$ & $\begin{array}{l}\text { ECP60MDF VTZ } \\
\text { ECP10SDF }\end{array}$ \\
\hline
\end{tabular}

TABLE I. The ECPs and basis sets used for the benchmark studies.

\footnotetext{
a The Dolg-Stoll small-core (DS-SC), Dolg-Stoll large-core (DS-LC), Trail-Needs (TN) ECPs and the corresponding basis sets are available on the following websites: http://www.tc.uni-koeln.de/PP/clickpse.en.html and https://vallico.net/casinoqmc/pplib/. In the notation ECP $n \mathrm{M}(\mathrm{S}) \mathrm{DF}$, $n=$ number of core electrons which are replaced by the pseudopotential, $\mathrm{M}(\mathrm{S}) \mathrm{DF}=$ multi(single)-electron fit, fully relativistic.
} 


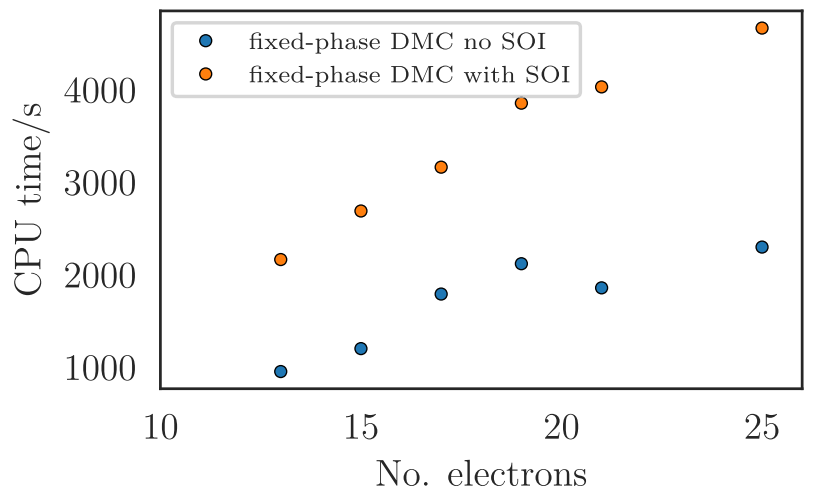

FIG. 8. CPU times needed for performing fixed-phase DMC on 1 core (16 nodes) for the same number of blocks, with and without the SOI part computed.

where coefficient $c$ is real, and the Slater determinants $D_{1}$ and $D_{2}$ are given by

$$
\begin{gathered}
D_{1}=\operatorname{Det}\left(\begin{array}{l}
\uparrow: 1,2,3,4,5,6,7,8,9,10,12,13 \\
\downarrow: 1,2,3,4,5,6,7,8,9,10,12,14
\end{array}\right), \\
D_{2}=\operatorname{Det}\left(\begin{array}{l}
\uparrow: 1,2,3,4,5,6,7,8,9,10,12,14 \\
\downarrow: 1,2,3,4,5,6,7,8,9,10,12,13
\end{array}\right) .
\end{gathered}
$$

The orbitals labeled by 13 and 14 are the highest-occupied and the lowest-unoccupied orbitals at $K$. They are annotated using the irreducible representations $A^{\prime \prime}$ and $A_{1}^{\prime}$ in Fig. 3 on the band structure plot. We thereby vary the coefficient $c$ from -1.0 to 1.0 and multiply the total wave function by a threebody Jastrow factor.

We project out the ground-state wave function of these trial wave functions using fixed-phase diffusion Monte Carlo, by propagating the wave function according to

$$
\begin{aligned}
\Psi(\mathbf{R}, \tau) & =\int G\left(\mathbf{R} \leftarrow \mathbf{R}^{\prime} ; \tau\right) \Psi_{T}\left(\mathbf{R}^{\prime}\right) d \mathbf{R}^{\prime} \\
& =\left\langle\mathbf{R}\left|\exp \left[-\tau\left(\widehat{H}_{\text {sca-rel }}-E_{T}\right)\right]\right| \Psi_{T}\right\rangle .
\end{aligned}
$$

One can easily show that the lowest-energy eigenstate of the Hamiltonian $\widehat{H}_{\text {sca-rel }}$ will be projected out when the wave function above is evolved for a long time. We apply the fixed-phase approximation for complex wave functions to circumvent the sign problem. With this approximation, the final fixed-phase wave function has the same nodal structure with that of the trial wave function, i.e., $D_{1}+i c D_{2}$. Thus, if we know that $D_{1}+i c D_{2}$ are the wave functions with the same scalar-relativistic energies, but differ only in their angular momenta, their fixed-phase wave functions should lie on a manifold that can be well described using a model Hamiltonian with the scale-separated scalar-relativistic energy and spin-orbit energy, i.e., $\widehat{H}_{\text {rel }}^{\text {eff }}=E_{0}+\widehat{H}_{\text {SOI- }}^{\text {eff }}$

\section{APPENDIX C: EFFECT OF ELECTRON CORRELATION ON THE SPIN-ORBIT INTERACTION STRENGTH}

For the Sn atom, where there are two electrons in the $p$ manifold, we also observe how the SOI energy is changed by including the Jastrow factors in the wave functions. Figure 6 shows the SOI energies versus the spin-orbit descriptor values for Sn, calculated using VMC, with single Slater determinants (labeled by HF) and Slater determinants multiplied by the same three-body Jastrow factor (VMC-SJ). One can see that in this system, adding in the Jastrow factor could change the spin-orbit interaction energy for the sampled wave function up to $0.021(2) \mathrm{eV}$.

\section{APPENDIX D: BASIS-SET DEPENDENCE OF THE SPIN-ORBIT SPLITTINGS}

In order to investigate whether the calculated spin-orbit splittings depend on the basis sets used to expand the singleparticle orbitals, we plot the normalized distribution of the fixed-phase DMC extrapolated estimator values (see Fig. 7) using different available basis sets [typically including up to double $\zeta$ (VDZ), triple $\zeta$ (VTZ), quadruple $\zeta$ (VQZ), and quintuple $\zeta$ (V5Z) functions] provided in Refs. [37,39,40]. The variation between different basis sets does not affect any conclusions in the paper.

All of the ECPs and basis sets used in the benchmark studies are listed in Table I. For each atom, we use the same Dolg-Stoll small-core ECP. In terms of the time-step extrapolation, we use $0.0025,0.005,0.01$, and 0.02 .

From Fig. 7, we see that most calculated probability distributions of the spin-orbit splittings are sharply peaked, indicating little dependence on basis sets.

TABLE II. The experimentally measured values and calculated values of the spin-orbit splittings for the $\mathrm{Ga}, \mathrm{In}, \mathrm{Ge}, \mathrm{Sn}, \mathrm{Br}$, and I atoms, using fixed-phase DMC, DFT (with PBE functional).

\begin{tabular}{ccccc}
\hline \hline Benchmark studies & & $\Delta_{\text {calc }}^{\mathrm{DFT}(\mathrm{PBE})} / \mathrm{eV}$ & $\Delta_{\text {calc }}^{\text {fixed-phase DMC }} / \mathrm{eV}$ (Trail-Needs ECP) & $\Delta_{\text {expt }} / \mathrm{eV}[49]$ \\
\hline \multirow{6}{*}{ Atoms } & $\mathrm{Ga}$ & 0.1152 & $0.0938(9)$ & 0.1024 \\
& $\mathrm{In}$ & 0.2949 & $0.2352(6)$ & 0.2743 \\
& $\mathrm{Ge}(J=1)$ & & $0.0542(3)$ & 0.0691 \\
& $\mathrm{Ge}(J=2)$ & 0.1856 & $0.1625(7)$ & 0.1748 \\
& $\mathrm{Sn}(J=1)$ & 0.4354 & $0.1346(9)$ & 0.2098 \\
& $\mathrm{Sn}(J=2)$ & 0.4947 & $0.407(3)$ & 0.4250 \\
$\mathrm{Br}$ & 0.9964 & $0.897(3)$ & 0.4569 \\
\hline
\end{tabular}




\section{APPENDIX E: COST OF FIXED-PHASE DMC CALCULATION OF THE SOI ENERGY}

Figure 8 shows the CPU times needed for performing fixed-phase DMC calculations with and without the SOI included (time step $=0.02,50$ blocks) for a different number of electrons, using the same ECP (Ga, Stuttgart-Stoll small core) on one core (16 nodes) on the Golub cluster (from the Illinois Campus Cluster Program). We can see that the calculational cost of fixed-phase DMC with SOI is approximately doubled when compared to that of the FN-DMC without SOI.

\section{APPENDIX F: DFT RESULTS OF THE SPIN-ORBIT SPLITTINGS FOR THE ATOMS (GA, IN, GE, SN, BR, I)}

In Table II, we present the results of the spin-orbit splittings calculated using DFT (with PBE functional [45]). The DFT calculations are performed using the open-source program QUANTUM ESPRESSO [53,54]. To compare our results with the DFT ones, we also included the results calculated using our method as well as the experimentally measured values.
[1] D. Pesin and L. Balents, Nat. Phys. 6, 376 (2010).

[2] W. Witczak-Krempa, G. Chen, Y. B. Kim, and L. Balents, Annu. Rev. Condens. Matter Phys. 5, 57 (2014).

[3] C. L. Kane and E. J. Mele, Phys. Rev. Lett. 95, 226801 (2005).

[4] B. A. Bernevig, T. L. Hughes, and S.-C. Zhang, Science 314, 1757 (2006).

[5] X.-L. Qi and S.-C. Zhang, Rev. Mod. Phys. 83, 1057 (2011).

[6] B. Kim, H. Ohsumi, T. Komesu, S. Sakai, T. Morita, H. Takagi, and T.-h. Arima, Science 323, 1329 (2009).

[7] X. Wan, A. M. Turner, A. Vishwanath, and S. Y. Savrasov, Phys. Rev. B 83, 205101 (2011).

[8] Z. Alpichshev, F. Mahmood, G. Cao, and N. Gedik, Phys. Rev. Lett. 114, 017203 (2015).

[9] K. T. Law and P. A. Lee, Proc. Natl. Acad. Sci. USA 114, 6996 (2017).

[10] A. Go, W. Witczak-Krempa, G. S. Jeon, K. Park, and Y. B. Kim, Phys. Rev. Lett. 109, 066401 (2012).

[11] G. Chen, R. Pereira, and L. Balents, Phys. Rev. B 82, 174440 (2010).

[12] G. Chen and L. Balents, Phys. Rev. B 84, 094420 (2011).

[13] D. R. Yarkony, Int. Rev. Phys. Chem. 11, 195 (1992).

[14] M. Sjøvoll, O. Gropen, and J. Olsen, Theor. Chem. Acc. 97, 301 (1997).

[15] O. Christiansen, J. Gauss, and B. Schimmelpfennig, Phys. Chem. Chem. Phys. 2, 965 (2000).

[16] B. A. Heß, Relativistic Effects in Heavy-Element Chemistry and Physics (Wiley, Chichester, 2003).

[17] P. Rosenberg, H. Shi, and S. Zhang, J. Phys. Chem. Solids 128, 161 (2019).

[18] L. K. Wagner, Phys. Rev. B 92, 161116(R) (2015).

[19] J. Yu, L. K. Wagner, and E. Ertekin, J. Chem. Phys. 143, 224707 (2015).

[20] C. Mitra, J. T. Krogel, J. A. Santana, and F. A. Reboredo, J. Chem. Phys. 143, 164710 (2015).

[21] J. Yu, L. K. Wagner, and E. Ertekin, Phys. Rev. B 95, 075209 (2017).

[22] A. Ambrosetti, P. L. Silvestrelli, F. Toigo, L. Mitas, and F. Pederiva, Phys. Rev. B 85, 045115 (2012).

[23] C. A. Melton, M. Zhu, S. Guo, A. Ambrosetti, F. Pederiva, and L. Mitas, Phys. Rev. A 93, 042502 (2016).

[24] C. A. Melton, M. C. Bennett, and L. Mitas, J. Chem. Phys. 144, 244113 (2016).

[25] A. Ambrosetti, F. Pederiva, E. Lipparini, and S. Gandolfi, Phys. Rev. B 80, 125306 (2009).

[26] A. Ambrosetti, J. M. Escartin, E. Lipparini, and F. Pederiva, Europhys. Lett. 94, 27004 (2011).
[27] A. Ambrosetti, F. Pederiva, and E. Lipparini, Phys. Rev. B 83, 155301 (2011).

[28] W. Zhao, Z. Ghorannevis, L. Chu, M. Toh, C. Kloc, P.-H. Tan, and G. Eda, ACS Nano 7, 791 (2013).

[29] H. J. Changlani, H. Zheng, and L. K. Wagner, J. Chem. Phys. 143, 102814 (2015).

[30] H. Zheng, H. J. Changlani, K. T. Williams, B. Busemeyer, and L. K. Wagner, Front. Phys. 6, 43 (2018).

[31] L. K. Wagner, M. Bajdich, and L. Mitas, J. Comput. Phys. 228, 3390 (2009).

[32] M. Casula, Phys. Rev. B 74, 161102(R) (2006).

[33] L. K. Wagner and J. C. Grossman, Phys. Rev. Lett. 104, 210201 (2010).

[34] W. M. C. Foulkes, L. Mitas, R. J. Needs, and G. Rajagopal, Rev. Mod. Phys. 73, 33 (2001).

[35] M. W. Schmidt, K. K. Baldridge, J. A. Boatz, S. T. Elbert, M. S. Gordon, J. H. Jensen, S. Koseki, N. Matsunaga, K. A. Nguyen, S. Su et al., J. Comput. Chem. 14, 1347 (1993).

[36] M. S. Gordon and M. W. Schmidt, in Theory and Applications of Computational Chemistry, edited by C. E. Dykstra, G. Frenking, K. S. Kim, and G. E. Scuseria (Elsevier, Amsterdam, 2005), pp. 1167-1189.

[37] B. Metz, H. Stoll, and M. Dolg, J. Chem. Phys. 113, 2563 (2000).

[38] H. Stoll, B. Metz, and M. Dolg, J. Comput. Chem. 23, 767 (2002).

[39] K. A. Peterson, D. Figgen, E. Goll, H. Stoll, and M. Dolg, J. Chem. Phys. 119, 11113 (2003).

[40] K. A. Peterson, B. C. Shepler, D. Figgen, and H. Stoll, J. Phys. Chem. A 110, 13877 (2006).

[41] J. R. Trail and R. J. Needs, J. Chem. Phys. 122, 174109 (2005).

[42] J. R. Trail and R. J. Needs, J. Chem. Phys. 122, 014112 (2005).

[43] R. Dovesi, A. Erba, R. Orlando, C. M. Zicovich-Wilson, B. Civalleri, L. Maschio, M. Rérat, S. Casassa, J. Baima, S. Salustro, and B. Kirtman, WIREs: Comput. Mol. Sci. 8, e1360 (2018).

[44] R. Dovesi, V. R. Saunders, C. Roetti, R. Orlando, C. M. Zicovich-Wilson, F. Pascale, B. Civalleri, K. Doll, N. M. Harrison, I. J. Bush, P. D’Arco, M. Llunell, M. Causà, Y. Noël, L. Maschio, A. Erba, M. Rérat, and S. Casassa, CRYSTAL17 User's Manual (University of Torino, Torino, 2017).

[45] J. P. Perdew, K. Burke, and M. Ernzerhof, Phys. Rev. Lett. 77, 3865 (1996).

[46] D. Figgen, K. A. Peterson, M. Dolg, and H. Stoll, J. Chem. Phys. 130, 164108 (2009). 
[47] G. Igel-Mann, H. Stoll, and H. Preuss, Mol. Phys. 65, 1321 (1988).

[48] L. K. Wagner and L. Mitas, J. Chem. Phys. 126, 034105 (2007).

[49] Y. Ralchenko, A. E. Kramida, J. Reader et al., NIST Atomic Spectra Database (version 3.1.5) (unpublished).

[50] W. Humphrey, A. Dalke, and K. Schulten, J. Mol. Graph. 14, 33 (1996).

[51] J. Kang, S. Tongay, J. Zhou, J. Li, and J. Wu, Appl. Phys. Lett. 102, 012111 (2013)

[52] M. Dolg and X. Cao, Chem. Rev. 112, 403 (2011).

[53] P. Giannozzi, S. Baroni, N. Bonini, M. Calandra, R. Car, C. Cavazzoni, D. Ceresoli, G. L. Chiarotti, M. Cococcioni, I. Dabo, A. D. Corso, S. de Gironcoli, S. Fabris, G. Fratesi, R. Gebauer, U. Gerstmann, C. Gougoussis, A. Kokalj, M. Lazzeri, L. Martin-Samos, N. Marzari, F. Mauri, R. Mazzarello, S.
Paolini, A. Pasquarello, L. Paulatto, C. Sbraccia, S. Scandolo, G. Sclauzero, A. P. Seitsonen, A. Smogunov, P. Umari, and R. M. Wentzcovitch, J. Phys.: Condens. Matter 21, 395502 (2009).

[54] P. Giannozzi, O. Andreussi, T. Brumme, O. Bunau, M. B. Nardelli, M. Calandra, R. Car, C. Cavazzoni, D. Ceresoli, M. Cococcioni, N. Colonna, I. Carnimeo, A. D. Corso, S. de Gironcoli, P. Delugas, R. A. DiStasio, A. Ferretti, A. Floris, G. Fratesi, G. Fugallo, R. Gebauer, U. Gerstmann, F. Giustino, T. Gorni, J. Jia, M. Kawamura, H.-Y. Ko, A. Kokalj, E. Küçükbenli, M. Lazzeri, M. Marsili, N. Marzari, F. Mauri, N. L. Nguyen, H.-V. Nguyen, A. O. de-la Roza, L. Paulatto, S. Poncé, D. Rocca, R. Sabatini, B. Santra, M. Schlipf, A. P. Seitsonen, A. Smogunov, I. Timrov, T. Thonhauser, P. Umari, N. Vast, X. Wu, and S. Baroni, J. Phys.: Condens. Matter 29, 465901 (2017). 